\title{
Spotlight on inhaled ciprofloxacin and its potential in the treatment of non-cystic fibrosis bronchiectasis
}

This article was published in the following Dove Press journal:

Drug Design, Development and Therapy

\author{
Stamatia Chorepsima ${ }^{1,2}$ \\ Konstantinos S Kechagias' \\ Georgios Kalimeris ${ }^{1,2}$ \\ Nikolaos A Triarides ${ }^{1,2}$ \\ Matthew E Falagas ${ }^{1-3}$ \\ 'Alfa Institute of Biomedical Sciences \\ (AIBS), Athens, Greece; ' 2 Department \\ of Medicine, Henry Dunant Hospital \\ Center, Athens, Greece; ${ }^{3}$ Department \\ of Medicine, Tufts University School of \\ Medicine, Boston, MA, USA
}

Correspondence: Matthew E Falagas Alfa Institute of Biomedical Sciences (AIBS), 9 Neapoleos Street, I5I 23 Marousi, Athens, Greece

Tel +302106839600

$\mathrm{Fax}+302106839605$

Email m.falagas@aibs.gr

\begin{abstract}
Non-cystic fibrosis bronchiectasis (NCFB) is a severe chronic illness characterized by irreversible dilation of airways and thickening of bronchial walls, chronic inflammation, repeated infections, and progressive obstruction of the airways. In contrast to cystic fibrosis bronchiectasis (CFB), which is a well-defined genetic disorder, NCFB is a heterogeneous disease caused by many different medical entities. Inhaled antibiotics are effective for patients with CFB, but their efficacy in NCFB has not been proven. The main pathogens involved in the colonization of patients with bronchiectasis are Haemophilus influenza, Moraxella catarrhalis, Staphylococcus aureus, and Pseudomonas aeruginosa. The latter is associated with increased morbidity and mortality. In addition, in NCFB, P. aeruginosa strains are frequently more resistant than those in CFB. At present, there are no approved inhaled antibiotic therapies for NCFB patients. Inhaled ciprofloxacin has been under investigation in the last few years. In two phase II randomized, double-blind, placebo-controlled trials, the use of inhaled ciprofloxacin was significantly associated with reduction in sputum bacterial density and greater eradication rates. In four phase III randomized, double-blind, placebo-controlled trials, the results regarding the time of the first exacerbation and the rate of exacerbations were inconsistent. Specifically, ORBIT-4 and RESPIRE-1 trials showed clinical benefit (prolongation of the time of the first exacerbation and reduced rate of exacerbations in the treatment group compared to the placebo group), whereas the ORBIT-3 and RESPIRE-2 failed to achieve their primary endpoints. The RESPIRE-1 was the first trial that examined the 14-days on/off course separate from the standard 28-days on/off regimen, which is based on CFB protocol treatments. The current data on the efficacy of inhaled ciprofloxacin are encouraging, but further evaluation is needed to determine the appropriate target group and the ideal duration of treatment.
\end{abstract}

Keywords: aerosolized antibiotics, ciprofloxacin, bronchiectasis, fluoroquinolone

\section{Introduction}

Non-cystic fibrosis bronchiectasis (NCFB) is an important health issue that is increasingly common and related to a considerably high mortality. ${ }^{1}$ It has higher incidence in older patients and females. The increased incidence of NCFB during the last decades is partially attributable to early diagnosis with the use of high-resolution spiral-computed tomography.

The main causes of NCFB include infections, bronchial obstruction, allergic bronchopulmonary aspergillosis, immunodeficiency states, connective tissue disorders, idiopathic inflammatory disorders, and autoimmune diseases. The most common cause in the literature is post-infectious, although no underlying cause is identified 
in a substantial percentage of patients. ${ }^{2}$ There is frequent, and often underestimated, coexistence of NCFB with COPD. ${ }^{2,3}$

\section{Inhaled antibiotics for patients with bronchiectasis}

Treatment for NCFB has been based on the general principles of management of cystic fibrosis bronchiectasis (CFB), with inhaled antibiotics being an adjunctive part of the treatment. ${ }^{4,5}$ The main advantages of inhaled antibiotics are the maximization of drug delivery to the site of infection and the protection from systemic adverse events. ${ }^{6}$ Inhaled antibiotics have been used for prevention and treatment for patients with tracheobronchitis, especially in the intensive care unit. Several original investigations and meta-analyses of clinical trials have suggested the potential of antibiotics, such as colistin, for the prevention and treatment of lower respiratory tract infections in hospitalized patients. ${ }^{7-9}$

There are commercially available inhaled antibiotics for patients with cystic fibrosis and colonization with Pseudomonas aeruginosa: two US Food and Drug Administration (FDA)-approved drugs, tobramycin (both as solution and dry powder) and aztreonam (solution), and two European Medicines Agency (EMA)-approved drugs, colistimethate (dry powder) and levofloxacin (solution). ${ }^{10-13} P$. aeruginosa infection is related to a three-fold increase in mortality risk and a seven-fold increase in hospitalization risk. ${ }^{4}$ According to current data, eradication of $P$. aeruginosa leads to better clinical outcomes. The use of intravenous antibiotics in combination with inhaled antibiotics achieves greater rates of $P$. aeruginosa clearance, compared to monotherapy with intravenous antibiotics. ${ }^{14}$ However, despite the similarities between CFB and NCFB in terms of clinical presentation and microbiology, the clinical benefit of the use of inhaled antibiotics in NCFB, in contrast to that for CFB, is not well established. ${ }^{15}$ Inhaled antibiotics that have been studied in patients with NCFB include tobramycin, gentamicin, amikacin, aztreonam, colistin, levofloxacin, and ciprofloxacin. A meta-analysis of eight randomized trials showed that inhaled antibiotics were more effective than placebo or symptomatic treatment in the eradication of bacteria from sputum, in the reduction of sputum bacterial load, and in the prevention of acute exacerbations. However, neither the risk of unscheduled hospitalizations nor the health-related quality of life (QoL) were improved. ${ }^{16-18}$

To date, there are no approved inhaled antibiotic therapies for patients with NCFB. The first international guidelines for adult bronchiectasis were published in September 2017 by the European Respiratory Society. Remarkably, most of treatment recommendations were based on low or very low quality of evidence, except for pulmonary rehabilitation which was supported by high-quality evidence. ${ }^{19}$

\section{Ciprofloxacin}

Ciprofloxacin is a second-generation fluoroquinolone, a broad-spectrum antibacterial with excellent activity against pathogens relevant to NCFB, such as $P$. aeruginosa, available in oral and intravenous formulations. The pharmacokinetics and the antibacterial spectrum of ciprofloxacin make it a promising option for NCFB treatment. ${ }^{20,21}$ The potential of achieving greater pulmonary concentrations with inhaled ciprofloxacin, compared to oral or intravenous administration, makes inhaled formulations of the antibiotic worthy of investigation.

Two formulations of inhaled ciprofloxacin - liposomal (CFI, ciprofloxacin for inhalation) and dry-powder for inhalation (DPI) - have been under investigation for their efficacy and safety in the treatment of NCFB patients. ${ }^{3}$ The pulmonary deposition of DPI ciprofloxacin was assessed by quantitative scintigraphy in a phase I study that included 24 participants (six patients with $\mathrm{NCFB}$, six with COPD, and 12 healthy volunteers). The results showed high and reproducible lung deposition (mean relative to nominal dose $53 \% \pm 11 \%$, range $38 \%-64 \%$ for NCFB patients; $51 \% \pm 10 \%$, range $34 \%-61 \%$ for COPD patients; and $51 \% \pm 7 \%$, range $40 \%-64 \%$ for healthy volunteers) and low-systemic exposure in all participants. ${ }^{22}$

The objective of this review was to evaluate the current data on the efficacy and safety of inhaled ciprofloxacin in NCFB patients.

\section{Clinical trials: current data}

The characteristics of the main clinical trials on the clinical efficacy of inhaled ciprofloxacin in NCFB patients are shown in Table 1.

A phase II, randomized, double-blind, placebo-controlled, multicenter study included 124 (60 in the DPI group and 64 in the placebo group) stable NCFB patients with positive sputum culture for respiratory pathogens, including $P$. aeruginosa. DPI or placebo were administered twice daily for 28 days, with 56 days of follow-up. At Day 29 (end of treatment), the results showed a statistically significant reduction in sputum bacterial density and greater eradication rates in the DPI group as compared to the placebo group $(-3.62$ $\log _{10}$ CFU.g ${ }^{-1}$ vs $-0.27 \log _{10}$ CFU.g ${ }^{-1}, P<0.001$ and $35 \%$ vs $8 \%, P=0.001$, respectively). ${ }^{20}$

Another phase II, 24-week, randomized, doublebind, placebo-controlled, trial - Once daily Respiratory 


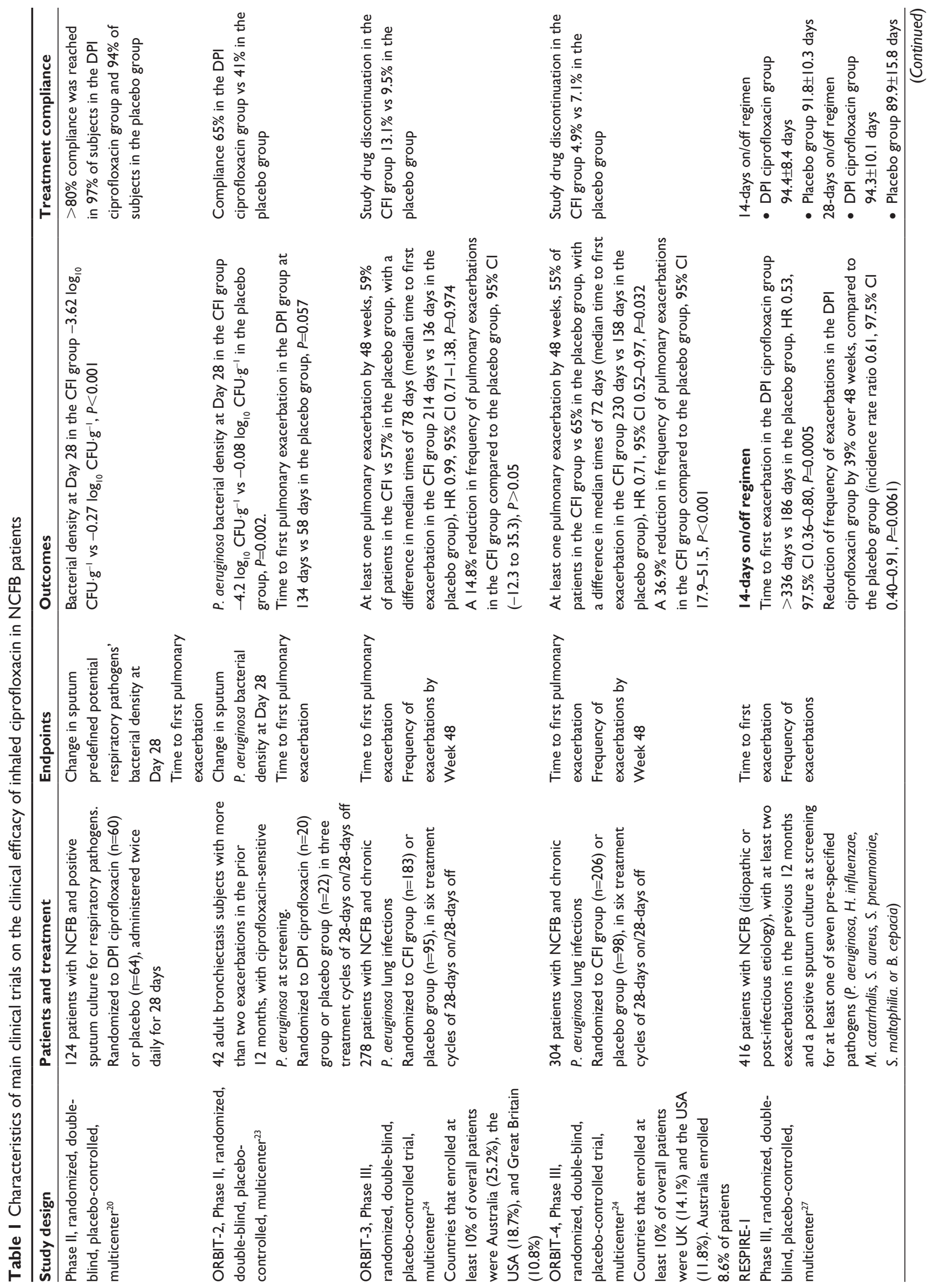




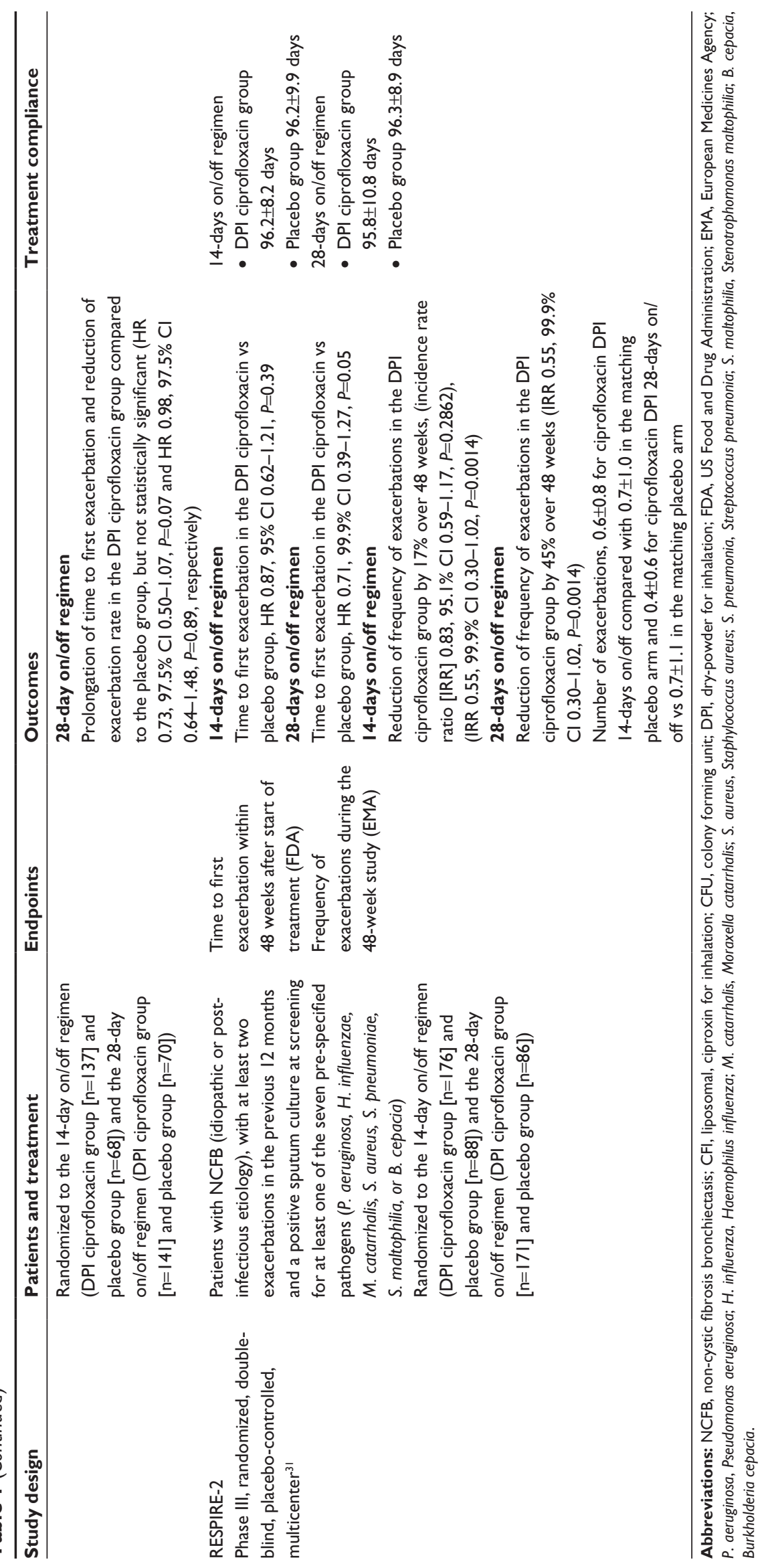


Bronchiectasis Inhalation Treatment (ORBIT-2) - examined the efficacy of dual-release CFI in 42 (20 in the CFI group and 22 in the placebo group) NCFB adults with two or more exacerbations in the last year and ciprofloxacin-sensitive $P$. aeruginosa at screening. CFI or placebo was administered once daily in three treatment cycles of 28-days on/28-days off. The results showed significant reductions in $P$. aeruginosa bacterial density on Day 28 in the CFI group, compared to the placebo group $\left(-4.2 \log _{10} \mathrm{CFU} \cdot \mathrm{g}^{-1}\right.$ vs $-0.08 \log _{10}$ CFU.g $\left.{ }^{-1}, P=0.002\right)$. Moreover, CFI delayed time to first pulmonary exacerbation in the studied population (134 days vs 58 days, $P=0.057$ ), although the study was not adequately powered for this endpoint. ${ }^{23}$

The phase III, 48-week multinational, randomized, doubleblind, placebo-controlled trials, ORBIT-3 (278 patients, $75.5 \%$ experienced $2-3$ pulmonary exacerbations in the 12 months prior to screening, $69.8 \%$ women, $30 \%$ men, mean age 65.1 [range 21-87] years, 183 in the CFI arm, 95 in the placebo arm) and ORBIT-4 (304 patients, 79.6\% experienced two pulmonary exacerbations in the 12 months prior to screening, $64.8 \%$ women, $35.2 \%$ men, mean age 63.6 [range 18-90] years, 206 in the CFI arm, 98 in the placebo arm) were designed to examine the efficacy of CFI in patients with NCFB and chronic $P$. aeruginosa lung infections. ${ }^{24,25}$ CFI or placebo were administered once daily for six cycles of 28-days on/off treatment. The primary endpoint of both studies was the increase in the median time to first pulmonary exacerbation and the secondary endpoints were the frequency of exacerbations, the number of severe (treatment with intravenous antibiotics or/and hospitalization) pulmonary exacerbations, and the change in the Respiratory Symptoms Domain Score for QoL. ${ }^{24}$

In ORBIT-4, the median time to first pulmonary exacerbation was 230 days in the CFI group vs 163 days in the placebo group $(P<0.05)$, and there was a $37 \%$ reduction in the frequency of pulmonary exacerbations in the CFI group $(P<0.001)$. In ORBIT-3, the median time to first pulmonary exacerbation was 221 days vs 136 days in the placebo group $(P=0.84)$, and there was a $13 \%$ reduction in the frequency of pulmonary exacerbations in the CFI group $(P=0.31)$. The pooled results showed a $27 \%$ reduction in the frequency of exacerbations (RR $0.73,95 \%$ CI $0.60-0.88, P<0.001$ ). No statistically significant differences were found between the two groups in either trial, with regard to the number of severe exacerbations or QoL assessed by the Respiratory Symptoms Domain Score. ${ }^{24}$

The baseline demographic characteristics of included patients in each study were balanced. However, in ORBIT-3, the percentage of patients with serious adverse events was higher than in ORBIT-4, which could partially be attributed to the indices of severity of the disease not measured, as well as to the differences in etiology of NCFB - information that was not collected by the researchers. Moreover, the rates of premature discontinuation were higher in the CFI group compared to the placebo group in ORBIT-3, whereas the opposite was noticed in ORBIT-4. ${ }^{24}$ The results of the ORBIT studies have not been fully published yet.

The RESPIRE program consists of two phase III, 48-week, randomized, multicenter placebo-controlled trials of the same design that examined the efficacy and safety of DPI in NCFB. They represent the largest clinical trial program conducted in NCFB. The two trials differed by the enrolling countries and the statistical analysis of the data. ${ }^{26}$ The RESPIRE-1 study included countries of Europe, North and South America, Australia, and Japan, whereas the RESPIRE-2 included countries of Asia and Eastern Europe. The primary endpoints of both studies were the efficacy of DPI in prolonging the time to the first exacerbation (analysis plan for FDA) and reducing the frequency of exacerbations (analysis plan for EMA). Secondary endpoints were microbiology parameters, QoL, and pulmonary function. In both trials, DPI or placebo was administered twice daily in cycles of 14-days on/off (12 cycles) or 28-days on/off ( for 48 weeks. The patients were followed for 8 weeks after the last dose. ${ }^{26,27}$

The RESPIRE-1 trial included 416 patients, randomized to the 14-days on/off regimen treatment (137 in the DPI group, 68 in the placebo group) or to the 28-days on/off regimen treatment (141 in the DPI group, 70 in the placebo group). The frequency of pulmonary exacerbations, in the DPI 14-days on/off group vs the matching placebo, was significantly reduced by $39 \%$ over 48 weeks (RR $0.61,95 \%$ CI $0.40-0.91, P=0.006$ ) and the time to first exacerbation was significantly prolonged in the DPI group vs placebo group (HR 0.53, 95\% CI 0.36-0.80, P<0.001). Furthermore, prolongation of time to the first exacerbation and reduction of exacerbations were seen in the DPI 28-day on/off group vs the matching placebo, but the results were not statistically significant (HR $0.73,95 \%$ CI $0.50-1.07, P=0.07$ and HR $0.98,95 \%$ CI $0.64-1.48, P=0.89$, respectively). ${ }^{27}$ In the RESPIRE-2 trial, no significant improvement was found with regard to the primary endpoints between the DPI and the placebo group, neither in the 14-days on/off nor in the 28-days on/off regimen treatment. The discrepancy between the results of the two studies reflects, possibly, the ethnic and geographic heterogeneity of the disease. ${ }^{28}$ 


\section{Adverse events}

The most common adverse events of inhaled antibiotics include bronchospasm, cough, throat irritation, and abnormal taste, whereas the current data for systemic toxicity are favorable. However, the major concern remains - the emergence of antibiotic-resistant pathogens. ${ }^{6}$

According to a meta-analysis of seven trials with 526 patients, the most common adverse event was bronchospasm, which occurred in $10 \%$ of patients treated with inhaled antibiotics and $2.3 \%$ in the control group (RR 2.96, 95\% CI $\left.1.30-6.73, P=0.01, I^{2} 0 \%\right){ }^{18}$ The results of the subgroup analysis showed that inhaled ciprofloxacin and colistin were not significantly related to the occurrence of this adverse event, contrary to inhaled aminoglycosides (ciprofloxacin, two trials with 166 patients, RR 1.07, 95\% CI 0.25-4.56, $P=0.93$; colistin, one trial with 144 patients, RR 4.86, $95 \%$ CI $0.58-40.59, P=0.14$; aminoglycosides, four trials with 216 patients, RR 4.78, 95\% CI 1.55-14.76, $P=0.007$ ).

\section{Development of microbial drug resistance}

The use of antibiotics for extended periods may decrease the symptoms and number of exacerbations, but it may result in the appearance of multi-drug resistant bacteria. There are data from recent studies which show that these bacteria have a considerable impact on exacerbations and communityacquired pneumonia in NCFB patients. ${ }^{29}$

$P$. aeruginosa, one of the main pathogens, and its isolates have a different phenotype compared to those of CFB. There is a high prevalence of hyper-mutable and wild strains with worse prognosis in NCFB than in CFB patients. According to the results of a recent meta-analysis that evaluated the efficacy and safety of inhaled antibiotics in NCFB, the risk of $P$. aeruginosa resistance did not differ between treatment and control groups (sub-analysis of five studies, RR 1.35, 95\% CI 0.61-2.96, $P=0.46) .{ }^{30}$ However, in the RESPIRE-1 trial, the data showed that, at any time point, at least one isolate from sputum with an elevated minimum inhibitory concentration (as defined by the systemic ciprofloxacin breakpoints) was found in 54\% of patients in the DPI 14-days on/off group and $53.9 \%$ of patients in the DPI 28 -days on/off group vs $36.2 \%$ of patients in the placebo group. ${ }^{31}$ In ORBIT-3 and ORBIT-4, the percentage increase of ciprofloxacin-resistant $P$. aeruginosa isolates in the CFI group by the end of the study was $23 \%$ and $31 \%$, respectively, whereas no percentage increase was observed in the placebo group. ${ }^{24}$

\section{Critical evaluation of the available data}

The ORBIT-4 showed clinical benefit (statistically significant prolongation of the time of the first exacerbation and lower rate of exacerbations) for the CFI group vs the placebo group, which was contrary to ORBIT-3 despite their almost identical design.

The same pattern was observed in the RESPIRE- 1 and RESPIRE-2 studies. The RESPIRE-2 trial did not meet the primary endpoints, whereas RESPIRE-1 showed significant reduced rate of exacerbations and increased time to the first exacerbation in the DPI group vs the placebo group in the 14-days on/off, but not in the 28-days on/off, regimen treatment. The RESPIRE-1 was the first trial that examined the 14-days on/off course apart from the standard 28-days on/off regimen, which is based on CFB protocol treatments. The results were encouraging and may be the beginning of a new dosing strategy in NCFB patients.

The long-term use of antibiotic treatment may be associated with the risk of emergence of ciprofloxacin-resistant pathogens. However, to appropriately evaluate this risk in clinical practice, a follow-up period longer than 8 weeks is essential. Moreover, an issue of great importance is the lack of evidence of QoL improvement of studied patients, which was a secondary endpoint of the conducted trials. The differences between CFB and NCFB with regard to the treatment response reflect the differences that exist in terms of pathophysiology. CFB is an inherited autosomal recessive disease, caused by mutations in the gene of cystic fibrosis that codes for a protein transmembrane conductance regulator (CFTR). The severity of the disease is different for each patient because of the poor penetrance of the mutated gene. However, the underlying mechanism is the same. Instead, NCFB is an heterogeneous disease, caused by multiple causes with many clinical phenotypes. ${ }^{21,32}$ Future clinical trials should stratify patients based on specific characteristics, such as the underlying cause, the severity of the disease, and the presence or absence of $P$. aeruginosa.

\section{Conclusion}

Despite the progress in the field of inhaled antibiotics in NCFB in the last few decades, there are three major unresolved issues that require further investigation: the appropriate target group of patients, the ideal duration of therapy, and the true associated risk of antibiotic resistance. More multicenter randomized-controlled studies are needed to answer these questions and clarify the most effective treatment of patients with this chronic illness. 


\section{Disclosure}

Matthew E Falagas participated in advisory boards of AstraZeneca, Infectopharm, Tetraphase, Shionogi, and Xellia; received lecture honoraria from Cipla, Merck, and Pfizer; and received research support from Shionogi, Tetraphase, and Helperby. The authors report no other conflicts of interest in this work.

\section{References}

1. Quint JK, Millett ER, Joshi M, et al. Changes in the incidence, prevalence and mortality of bronchiectasis in the UK from 2004 to 2013 : a population-based cohort study. Eur Respir J. 2016;47(1):186-193.

2. Hill AT, Pasteur M, Cornford C, Welham S, Bilton D. Primary care summary of the British Thoracic Society Guideline on the management of non-cystic fibrosis bronchiectasis. Prim Care Respir J. 2011;20(2): 135-140.

3. Fjaellegaard K, Sin MD, Browatzki A, Ulrik CS. Antibiotic therapy for stable non-CF bronchiectasis in adults - A systematic review. Chron Respir Dis. 2017;14(2):174-186.

4. Polverino E, Goeminne PC, Mcdonnell MJ, et al. European Respiratory Society guidelines for the management of adult bronchiectasis. Eur Respir J. 2017;50(3):1700629.

5. Barker AF, O'Donnell AE, Flume P, et al. Aztreonam for inhalation solution in patients with non-cystic fibrosis bronchiectasis (AIR-BX1 and AIR-BX2): two randomised double-blind, placebo-controlled phase 3 trials. Lancet Respir Med. 2014;2(9):738-749.

6. Quon BS, Goss CH, Ramsey BW. Inhaled antibiotics for lower airway infections. Ann Am Thorac Soc. 2014;11(3):425-434.

7. Falagas ME, Trigkidis KK, Vardakas KZ. Inhaled antibiotics beyond aminoglycosides, polymyxins and aztreonam: A systematic review. Int J Antimicrob Agents. 2015;45(3):221-233.

8. Korbila IP, Michalopoulos A, Rafailidis PI, Nikita D, Samonis G, Falagas ME. Inhaled colistin as adjunctive therapy to intravenous colistin for the treatment of microbiologically documented ventilatorassociated pneumonia: a comparative cohort study. Clin Microbiol Infect. 2010;16(8):1230-1236.

9. Ioannidou E, Siempos II, Falagas ME. Administration of antimicrobials via the respiratory tract for the treatment of patients with nosocomial pneumonia: a meta-analysis. J Antimicrob Chemother. 2007;60(6): 1216-1226.

10. Ramsey BW, Dorkin HL, Eisenberg JD, et al. Efficacy of aerosolized tobramycin in patients with cystic fibrosis. NEngl J Med. 1993;328(24): 1740-1746.

11. Hazinski TA. Intermittent administration of inhaled tobramycin in patients with cystic fibrosis. J Pediatr. 1999;135(1):130.

12. Daniels LM, Juliano J, Marx A, Weber DJ. Inhaled Antibiotics for Hospital-Acquired and Ventilator-Associated Pneumonia. Clin Infect Dis. 2017;64(3):386-387.

13. Elborn JS, Vataire AL, Fukushima A, et al. Comparison of Inhaled Antibiotics for the Treatment of Chronic Pseudomonas aeruginosa Lung Infection in Patients With Cystic Fibrosis: Systematic Literature Review and Network Meta-analysis. Clin Ther. 2016;38(10):2204-2226.

14. Orriols R, Roig J, Ferrer J, et al. Inhaled antibiotic therapy in non-cystic fibrosis patients with bronchiectasis and chronic bronchial infection by Pseudomonas aeruginosa. Respir Med. 1999;93(7):476-480.

15. Dhand R. The Rationale and Evidence for Use of Inhaled Antibiotics to Control Pseudomonas aeruginosa Infection in Non-cystic Fibrosis Bronchiectasis. J Aerosol Med Pulm Drug Deliv. 2018;31(3):121-138.
16. Barker AF, Couch L, Fiel SB, et al. Tobramycin solution for inhalation reduces sputum Pseudomonas aeruginosa density in bronchiectasis. Am J Respir Crit Care Med. 2000;162(2 Pt 1):481-485.

17. Drobnic ME, Suñé P, Montoro JB, Ferrer A, Orriols R. Inhaled tobramycin in non-cystic fibrosis patients with bronchiectasis and chronic bronchial infection with Pseudomonas aeruginosa. Ann Pharmacother. 2005;39(1):39-44

18. Brodt AM, Stovold E, Zhang L. Inhaled antibiotics for stable non-cystic fibrosis bronchiectasis: a systematic review. Eur Respir J. 2014;44(2): 382-393.

19. Chalmers JD, Chotirmall SH. Bronchiectasis: new therapies and new perspectives. Lancet Respir Med. 2018;6(9):715-726.

20. Wilson R, Welte T, Polverino E, et al. Ciprofloxacin dry powder for inhalation in non-cystic fibrosis bronchiectasis: a phase II randomised study. Eur Respir J. 2013;41(5):1107-1115.

21. Justo JA, Danziger LH, Gotfried MH. Efficacy of inhaled ciprofloxacin in the management of non-cystic fibrosis bronchiectasis. Ther Adv Respir Dis. 2013;7(5):272-287.

22. Stass H, Nagelschmitz J, Kappeler D, Sommerer K, Kietzig C, Weimann B. Ciprofloxacin Dry Powder for Inhalation in Patients with Non-Cystic Fibrosis Bronchiectasis or Chronic Obstructive Pulmonary Disease, and in Healthy Volunteers. J Aerosol Med Pulm Drug Deliv. 2017;30(1): 53-63.

23. Serisier DJ, Bilton D, de Soyza A, et al. Inhaled, dual release liposomal ciprofloxacin in non-cystic fibrosis bronchiectasis (ORBIT-2): a randomised, double-blind, placebo-controlled trial. Thorax. 2013;68(9): $812-817$.

24. US FDA. FDA Briefing Document; Ciprofloxacin Dispersion for Inhalation (DI); Meeting of the Antimicrobial Drugs Advisory Committee (AMDAC); January 11, 2018. Available from: https://www.fda.gov/ downloads/AdvisoryCommittees/CommitteesMeetingMaterials/Drugs/ Anti-InfectiveDrugsAdvisoryCommittee/UCM591746.pdf. Accessed July 17, 2018.

25. Charles Haworth AW, Froehlich J, O’Neal T, Davis A, Gonda I, O’Donnell A. Inhaled Liposomal Ciprofloxacin in Patients with Bronchiectasis and Chronic Pseudomonas Aeruginosa Infection: Results from Two Parallel Phase III Trials (ORBIT-3 and -4). 2017(Abstract).

26. Chotirmall SH, Chalmers JD. RESPIRE: breathing new life into bronchiectasis. Eur Respir J. 2018;51(1):1702444.

27. de Soyza A, Aksamit T, Bandel T-J, et al. RESPIRE 1: a phase III placebo-controlled randomised trial of ciprofloxacin dry powder for inhalation in non-cystic fibrosis bronchiectasis. Eur Respir J. 2018; 51(1):1702052.

28. Aksamit T, de Soyza A, Bandel T-J, et al. RESPIRE 2: a phase III placebo-controlled randomised trial of ciprofloxacin dry powder for inhalation in non-cystic fibrosis bronchiectasis. Eur Respir J. 2018; 51(1):1702053.

29. Sahuquillo Mj MA, Hernandez Cabezas A, Menéndez R. Non-cystic fibrosis bronchiectasis: The long road to multidrug resistant bacteria Community Acquir Infect. 2016;3:110-117.

30. Xu L, Zhang F, Du S, et al. Inhaled antibiotics in non-cystic fibrosis bronchiectasis: A meta-analysis. Pharmazie. 2016;71(9):491-498.

31. Aksamit T, Bandel TJ, Criollo M, et al. The RESPIRE trials: Two phase III, randomized, multicentre, placebo-controlled trials of Ciprofloxacin Dry Powder for Inhalation (Ciprofloxacin DPI) in non-cystic fibrosis bronchiectasis. Contemp Clin Trials. 2017;58:78-85.

32. Mcshane PJ, Naureckas ET, Tino G, Strek ME. Non-cystic fibrosis bronchiectasis. Am J Respir Crit Care Med. 2013;188(6):647-656. 


\section{Publish your work in this journal}

Drug Design, Development and Therapy is an international, peerreviewed open-access journal that spans the spectrum of drug design and development through to clinical applications. Clinical outcomes, patient safety, and programs for the development and effective, safe, and sustained use of medicines are the features of the journal, which

has also been accepted for indexing on PubMed Central. The manuscript management system is completely online and includes a very quick and fair peer-review system, which is all easy to use. Visit http://www.dovepress.com/testimonials.php to read real quotes from published authors.

Submit your manuscript here: http://www.dovepress.com/drug-design-development-and-therapy-journal 